\title{
Characterization of Carbon Nanostructures in Al and Ag Covetic Alloys
}

\author{
H. M. Iftekhar Jaim ${ }^{1}$, Daniel P. Cole ${ }^{2}$, Lourdes G. Salamanca-Riba ${ }^{1 *}$ \\ ${ }^{1}$ Materials Science and Engineering Department, University of Maryland, College Park, MD 20742, USA \\ ${ }^{2}$ U.S. Army Research Laboratory, Aberdeen Proving Ground, MD 21005, USA \\ E-mail: riba@umd.edu
}

\begin{abstract}
Electrocharging assisted process is a method for the incorporation of carbon in molten metals under high electric current which results in the formation of networks of carbon nanostructures inside the metal matrix, and gives the new material improved mechanical, electrical and thermal properties. Alloys produced with this method are called covetics. In our previous works, different characterization techniques such X-ray photoelectron spectroscopy (XPS), Raman, X-ray diffraction (XRD), transmission electron microscopy (TEM) and electron energy loss spectroscopy (EELS) confirmed the presence of $\mathrm{sp}^{2}$ crystalline graphene nanoribbons and sheets in Ag and Al-covetic samples. Here, we report on detailed Raman mapping and characterization of Al-6061, Al-7075 and Ag covetics to further investigate the fraction of $\mathrm{sp}^{2} / \mathrm{sp}^{3}$ bonding, strain, defects, degree of oxidation, and crystalline sizes of the graphene nanoribbons. Gradual changes of strain are observed in regions with $\mathrm{sp}^{2}$ bonding and some degree of amorphous carbon is revealed by Raman scattering. Different degrees of oxidation of the carbon nanostructures with mostly $\mathrm{sp}^{2}$ bonding are evident by EELS spectrum imaging. Atomic force microscopy (AFM) and Kelvin probe force microscopy (KPFM) also reveal the presence of graphene ribbons and sheets with different lengths and conductivity.
\end{abstract}

\section{Introduction}

Carbon incorporation in bulk, thin films and nanostructures such as nanoparticles and nanowires is one of the major areas of research in condensed matter physics and materials science with the expectation of enhancing the electrical, thermal, and mechanical properties of materials used in electronic devices, thermal management, mechanical and structural applications [1]. These physical properties are dependent upon the structural form (crystalline or amorphous), density of defects, degree of oxidation, stacking order and orientation of various carbon nanostructures like graphene [2], graphite, fullerene [1], carbon nanotubes (CNT) [3], diamond and other allotropes of carbon. In particular, carbon addition in metals in the form of CNT or graphene sheets has an attractive prospect to take advantage of the superior mechanical, electrical and structural properties that $\mathrm{sp}^{2}$ bonding of carbon in these nanostructures provides in order to enhance the electrical and high strength properties of metals, and to improve the metals' resistance to oxidation. The unfavorable free energy changes in metals like Ag [4], $\mathrm{Au}$ [5] and $\mathrm{Cu}$ [6] limit the incorporation of carbon to ppm level under normal kinetics conditions in contrast to the high carbon content that is possible to incorporate in steel. In the last decade, novel processing procedures utilizing non-equilibrium kinetics like chemical vapor deposition (CVD) [7, 8], ball milling [9], plasma spray [10] and other techniques, have made possible the incorporation of carbon nanostructures like CNT and graphene in metals both in bulk and thin films resulting in 
hybrid materials with interesting material properties. However, laboratory based processing methods are often limited, and/or not suitable for mass production due to either multistep complexity or cost. More importantly, many of these techniques fail to retain the $\mathrm{sp}^{2}$ bonding of the carbon, essential for high electrical and thermal conductivities, or give rise to composites of the metal and carbon phases with weak bonding between the two components. Therefore, a simple single step processing for incorporation of carbon with $\mathrm{C}-\mathrm{C} \mathrm{sp}^{2}$ bonding and strong bonding between the metal and the carbon is desirable for large scale production and cost effectiveness. Aluminum alloys with carbon, in particular, are being considered as a possible replacement in power transmission lines if they exhibit higher conductivity and higher strength than the pure alloys.

Recently, Third Millennium Materials, LLC introduced a new process in which a very high current is applied to a molten metal like $\mathrm{Ag}, \mathrm{Al}, \mathrm{Cu}, \mathrm{Fe}, \mathrm{Au}$ and others while mixing and stirring particles of activated carbon in an inert gas environment. We call this process electrocharging assisted process since we believe that the applied current induces ionization of the carbon in the particles followed by polymerization which gives rise to the formation of graphitic structures inside the liquid metal. The produced samples, called covetics, have shown the presence of carbon nanostructures and are identified by the inventors of the process as $\mathrm{M} \mathrm{cv} \times \% \mathrm{C}(\mathrm{M}=\mathrm{Ag}$, $\mathrm{Al}, \mathrm{Cu}$, etc., is the metal host and $\mathrm{x}=$ nominal weight percentage of carbon added in the process). It is important to note that the actual concentration of carbon that incorporates in the lattice of the metal is lower than the nominal value. Nevertheless, $\mathrm{Al}$ alloys, $\mathrm{Cu}$ and $\mathrm{Ag}$ covetics have shown superior mechanical, electrical and thermal properties [11-14]. We have previously reported the presence of carbon nanostructures like graphene nanoribbons (GNR) and graphene sheets observed in TEM [11, 12]. These carbon nanostructures show primarily $\mathrm{sp}^{2} \mathrm{C}-\mathrm{C}$ bonding, and weak indication of Al-C bonding was confirmed by XPS in Al covetic samples [11]. Preliminary studies by EELS corroborated mostly $\mathrm{sp}^{2}$ bonding with small $\mathrm{sp}^{3}$ mixtures and different stages of oxidation in these alloys. In another case, thin films (10-30 nm thick) produced from a Cucovetic target with $5 \mathrm{wt} \% \mathrm{C}$ showed higher transmittance and higher resistance to oxidation than pure copper films of the same thickness indicating that copper covetic films could be used as transparent electrodes [15]. In this manuscript we perform a detailed study of the degree of $\mathrm{sp}^{2}$ and $\mathrm{sp}^{3}$ bonding, crystallite size of the graphitic structures and degree of strain in $\mathrm{Al}$ alloys and Ag covetics by combining Raman scattering, AFM/KPFM and EELS spectrum imaging from the covetic samples.

It is well known that the properties of the different carbon allotropes depend on whether the C-C bonding is $\mathrm{sp}^{2}$ or $\mathrm{sp}^{3}$, and even after 50 years of tremendous efforts, new features are found in carbon bonding unveiling fascinating avenues for research. Therefore, the analysis of the structure and chemical bonding of carbon nanostructures in covetics using versatile techniques such as XPS, XRD, AFM, Raman scattering, TEM, EELS, energy dispersive X-ray spectroscopy (EDS), and scanning electron microscopy (SEM) are of great importance. Among these techniques, Raman spectroscopy is one of the most powerful, non-destructive, inexpensive and fast techniques which has become ubiquitous for the analysis of carbon nanostructures $[16,17]$ since it can discern between single, double or more layers of graphene, carbon nanotubes, amorphous carbon, graphite and diamond. AFM in conjunction with KPFM is effective to find carbon phases in metal matrices by differentiating the potential contrast of the phases. EELS 
provides an opportunity to map carbon with bonding information alongside with other elements and shows the oxidation state of different elements.

In this manuscript, detailed materials characterization of the $\mathrm{C}-\mathrm{C}$ bonding and $\mathrm{C}$-metal bonding in Al-6061 cv 3\%, Al-7075 cv 5\%, Al-7075 cv 3\% and Ag cv 6\% is presented. To probe the very small carbon nanostructures, the role of $\mathrm{sp}^{2} / \mathrm{sp}^{3}$ bonding, oxidation, strain distribution, defect concentration, and the crystallite sizes of the graphitic structures, Raman mapping was carried out on covetic samples with different nominal carbon concentrations. EELS spectrum imaging and mapping of the different elements' edges were also acquired to probe the carbon nanostructures at a nanometer level and corroborate the Raman findings. EELS maps were analyzed by blind source separation technique to discriminate between the regions with $\mathrm{sp}^{2}$ and $\mathrm{sp}^{3}$ bonding. AFM and KPFM confirmed the presence of GNR and graphene sheets.

\section{Experimental Details}

Covetic samples prepared by applying a very high current to the molten metals and mixing particles of activated carbon of 10-50 $\mu \mathrm{m}$ size were obtained from Third Millennium Materials, LLC. The molten solution was stirred to get uniform mixing of carbon in the liquid, and Ar-gas was sprayed over the liquid surface to avoid oxidation. Electro-migration of carbon ions under the influence of the applied high current is believed to be the mechanism of carbon incorporation in the covetics process. Moreover, the high temperature along with the electric field gradient is likely to enable the activation energy for breaking/creating bonds, and also to contribute to faster kinetics. Al-6061 cv 3\%, Ag cv 6\%, and Al-7075 cv 3\% and 5\% were produced in the same manner, and small pieces of the samples were cut to investigate the carbon incorporation in the bulk of the samples. The cut pieces were mechanically polished with alumina papers of different grating starting from $12 \mu \mathrm{m}$ and finishing with $300 \mathrm{~nm}$ to obtain a smooth surface and to remove impurities from the cutting saw. Afterwards, samples were cleaned by sonication in acetone.

Raman mapping was performed using a confocal Raman microscope from Yvon Jobin LabRam ARAMIS with $532 \mathrm{~nm}$ laser (Nd: YAG source) using a 100X objective lens at 600 grating. The scanning area was $20 \mu \mathrm{m} \times 20 \mu \mathrm{m}$ with $1 \mu \mathrm{m}$ spacing in a square array of points. The laser spot size was around $700 \mathrm{~nm}$. Three scans were obtained from each point and averaged to avoid spikes and obtain a better signal/noise ratio. Maps were acquired in different areas of the sample at random, and areas that were representative of the whole surface were chosen. The setting parameters such as acquisition time, confocal aperture and slit sizes were kept constant for all of the $\mathrm{Al}$ and $\mathrm{Ag}$ sample sets to offset the variation effects arising from instrumental configuration. Duo-scan mode in the instrument averaged out the signal from the surrounding areas of each point enabling data acquisition from the whole $20 \mu \mathrm{m} \times 20 \mu \mathrm{m}$ area. The acquired data were analyzed, peak fitted and customized with Labspec, OriginPro and Matlab software packages. Fittings of the D and G-peaks, and the ratios of the integrated area under their peaks $\left(\mathrm{I}_{\mathrm{D}} / \mathrm{I}_{\mathrm{G}}\right)$ gave information on the defect concentration and crystallite size. For best fitting results, the G-peak, D-peak and amorphous carbon peaks were fitted with Breti-Wigner-Fano (BWF), Lorentzian and Gaussian curves, respectively [18]. Asymmetry of the G-peak matches BWF fitting in graphene/graphite and their derivatives which results from the interference effect between phonon spectra and electron-hole pair excitation spectra at the Dirac cone [19]. Disordered 
amorphous states are found to correspond well with Gaussian fitting whereas the crystalline/defective nature of the carbon D-peak is best fit by Lorentzian curves [18].

Scanning probe microscopy measurements were performed with an Asylum Research Cypher AFM. The platinum silicide-coated probes used in the study (PtSi-FM, Nanosensors) had resonance frequency, spring constant, and radius of curvature of approximately $75 \mathrm{kHz}, 5 \mathrm{Nm}^{-1}$, $25 \mathrm{~nm}$, respectively. Height, phase, and scanning KPFM maps were performed in AC mode with a scan rate of approximately $1 \mathrm{~Hz}$. In KPFM, the difference in work function between the tip and sample is measured in order to map relative surface potential. The KPFM technique was used in the present study to investigate variations in the physical and chemical properties of the covetic materials with respect to the control metals without carbon. KPFM is an attractive technique for local characterization of covetics because it allows for the possibility of distinguishing elemental variations that are not always possible with standard topography and phase mapping.

Elemental composition and bonding analysis were studied by EELS using a Gatan GIF Tridiem post column energy filter in a JEOL 2100 Field emission TEM. Different locations were mapped in scanning transmission electron microscopy (STEM) mode for rectangular areas under high angle annular dark field (HAADF) imaging for the elemental mapping of $\mathrm{Al}, \mathrm{Ag}, \mathrm{C}$ and $\mathrm{O}$. The pixel and scanning spot sizes were $1.5 \mathrm{~nm}$. Background subtraction of the EELS signal with a power law dependence was used to extract the $\mathrm{C}-\mathrm{K}$ edge in order to enhance details of the bonding and oxidation state of the carbon using Digital Micrograph software.

Further analysis of the EELS data was done with a Python-based open source software Hyperspy 0.8.4 [20] used for blind source separation (BSS) [21] of the signals acquired at each pixel. This signal processing technique is purely based on principal component analysis (PCA) [22] and machine learning without any prior conditioning or assumption unlike other instrumental software. BSS PCA identifies redundant signals based on their relative presences and results in the deconvolution of the main signals and noise. The software is designed specially to handle large data sets including spectrum data from EELS.

\section{Results}

\subsubsection{Raman mapping of Al-covetic samples}

The Raman study of pure Al-6061 showed no trace of carbon in the metal alloy besides the expected contamination from hydrocarbon on the surface[11]. In contrast, Figure $1 \mathrm{~B}$ presents a typical Raman spectrum of the Al-6061 covetic sample taken from one of the points marked in Figure 1 A clearly showing both $G$ and D-peaks. Peak deconvolution of the selected region comprising $\mathrm{G}$ and D-peaks is presented in Figure $1 \mathrm{C}$. The G- and D-peaks were fitted with BWF and Lorentzian peaks located at 1594 and $1348 \mathrm{~cm}^{-1}$, respectively. The Gaussian peak at 1518 $\mathrm{cm}^{-1}$ is probably due to amorphous carbon. The summed fitted data matches the experimental data very well. 
Some intensity variation of the G and D-peaks were observed during mapping of the Al-6061 covetic sample (Figure $2 \mathrm{~A}$ and B). G and D-peak positions and their FWHM maps are plotted in Figure $2 \mathrm{C}, \mathrm{D}, \mathrm{E}$ and $\mathrm{F}$, respectively. The regions with low intensity present blue shift of the Gpeaks (Figure 2 C) with lower FWHM (Figure 2 E) and red shift of the D-peaks (Figure 2 D) with lower FWHM (Figure $2 \mathrm{~F}$ ). Other than these regions, most of the area in Figure $2 \mathrm{C}$ shows uniform G-peak position at $\sim 1585 \mathrm{~cm}^{-1}$ corresponding to unstrained graphitic regions. However, the map of the D- peak position (Figure 2D) shows more variation centered mostly around $1350 \pm 10 \mathrm{~cm}^{-1}$.

A.

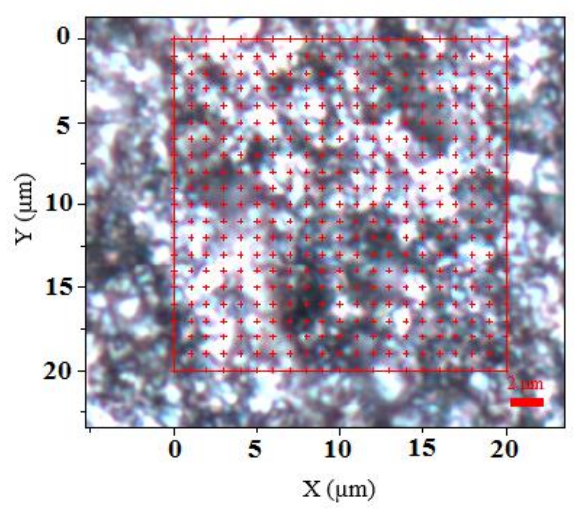

B.

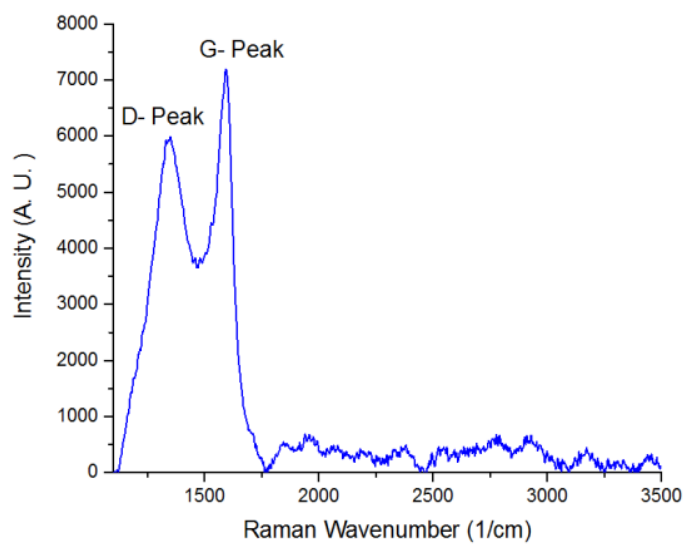

C.

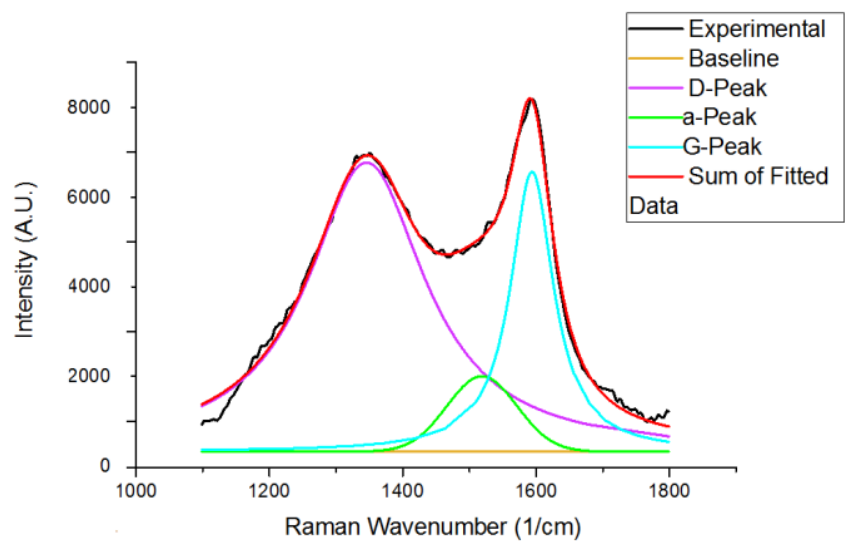

Figure 1: A. Optical image from Al-6061 covetic 3\% showing the scanned area of $20 \mu \mathrm{m} \times 20$ $\mu \mathrm{m}$ outlined by the red square. B. Raman spectrum of a point showing $\mathrm{G}$ and D peaks at 1594 $\mathrm{cm}^{-1}$ and $1348 \mathrm{~cm}^{-1}$, respectively with some minor peaks at high wavenumber regions resembling overlapping weak $2 G$ and $D+D^{\prime}$ regions. C. Peak fitting of the experimental data in $G$ and $D$ regions with BWF for G-peak, Gaussian for amorphous peak and Lorentzian for D-peak. In addition to the $\mathrm{G}$ and $\mathrm{D}$ peaks, a peak at $1518 \mathrm{~cm}^{-1}$ is indicative of the presence of amorphous carbon. The ratio of areas under the $\mathrm{D}$ and $\mathrm{G}$ peaks, $\mathrm{I}_{\mathrm{D}} / \mathrm{I}_{\mathrm{G}}$ is 2.4 . 

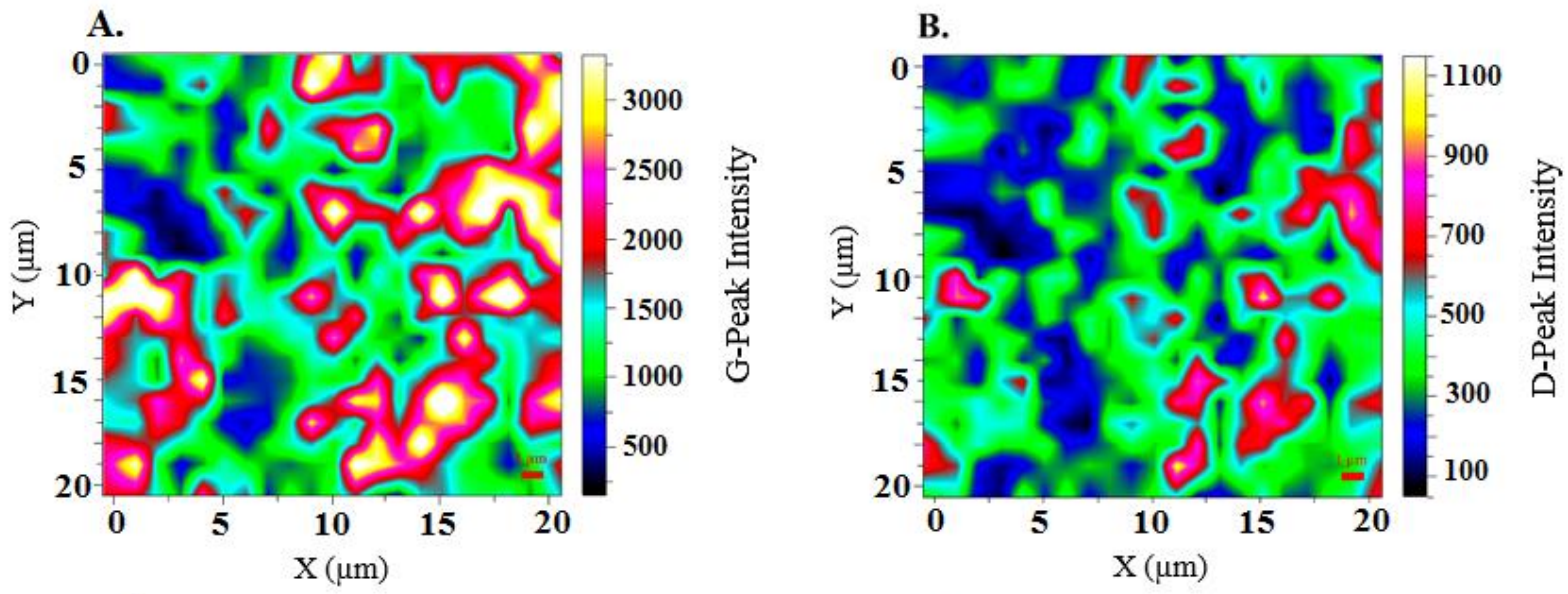

C.

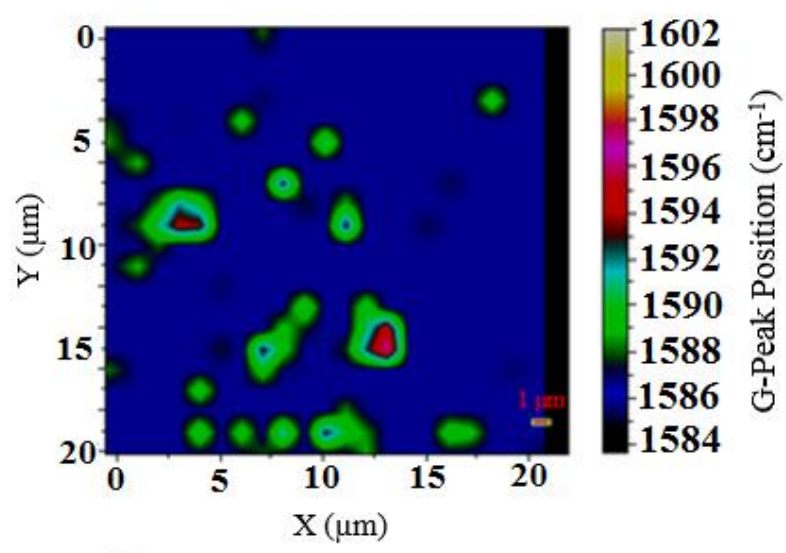

E.
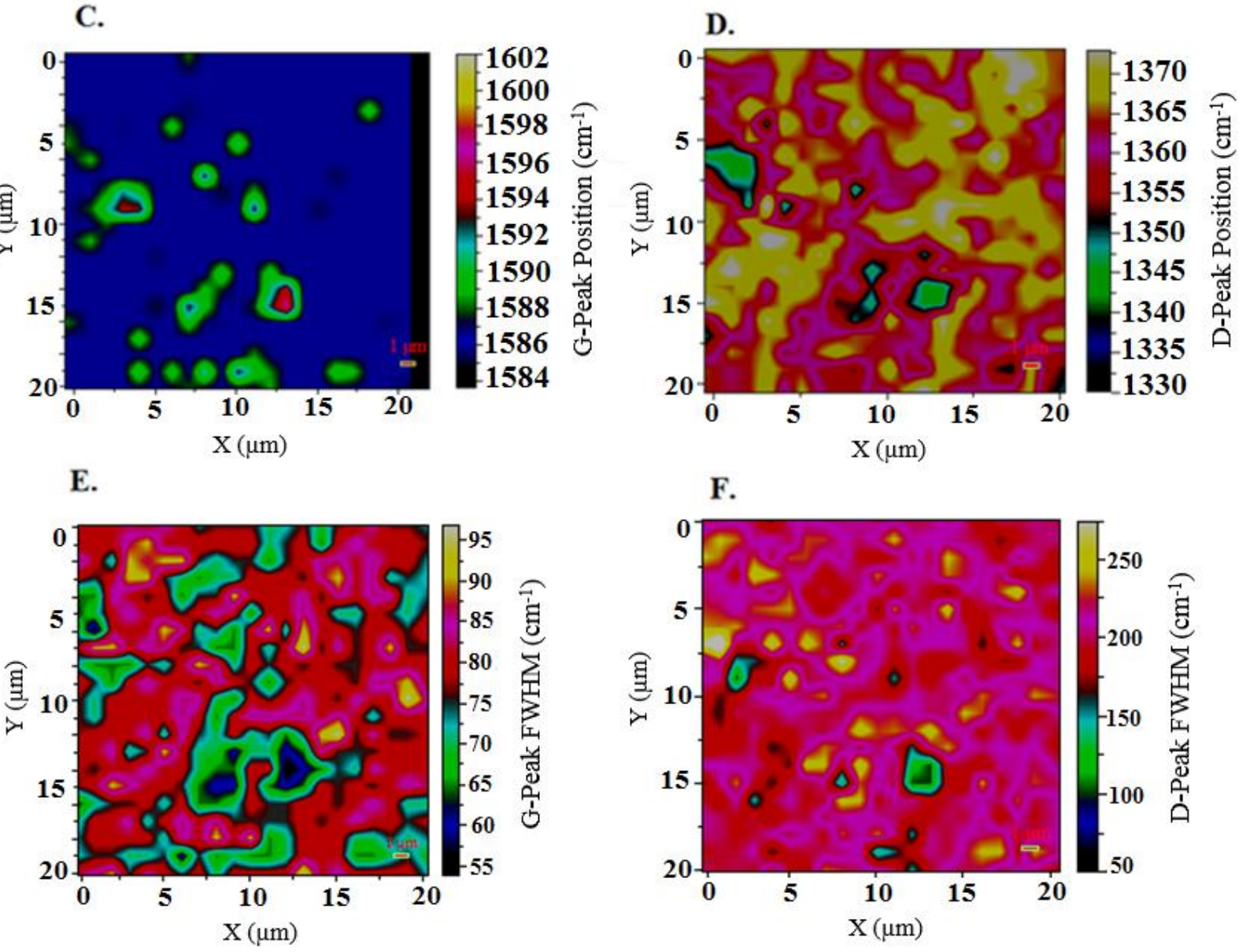

Figure 2: Raman mapping of $20 \mu \mathrm{m} \times 20 \mu \mathrm{m}$ area of the Al-6061 cv $3 \%$ in the red square of Figure 1 A. A. G-peak intensity, B. D-peak intensity. C. G-peak position. D. D-peak position. E. G-peak FWHM. F. D-peak FWHM. 
The crystallite size, $L_{a}$, obtained from the ratio of the intensities of the $G$ and $D$ peaks $\left(\mathrm{I}_{\mathrm{G}} / \mathrm{I}_{\mathrm{D}}\right)$ can be obtained using eq (1) [23].

$\mathrm{L}_{\mathrm{a}}(\mathrm{nm})=\left(2.4 \times 10^{-10}\right) \lambda^{4}$ laser $\left(\mathrm{I}_{\mathrm{G}} / \mathrm{I}_{\mathrm{D}}\right)$

where, $\lambda_{\text {laser }}$ is the wavelength of the laser used $(532 \mathrm{~nm})$. Statistical analysis of the integrated intensities of the $G$ and $D$ peaks was carried out over 37 randomly selected data points of the scanned area of Al-6061 cv $3 \%$ giving values of $L_{a}$ in the range 8 to $10 \mathrm{~nm}$.

The spectra from each point was used to correlate the FWHM and the peak positions. Just like the maps in Figure 2, Figure 3 corroborates that high G-peak and low D-peak positions correspond to regions with lower FWHM trends.

A.

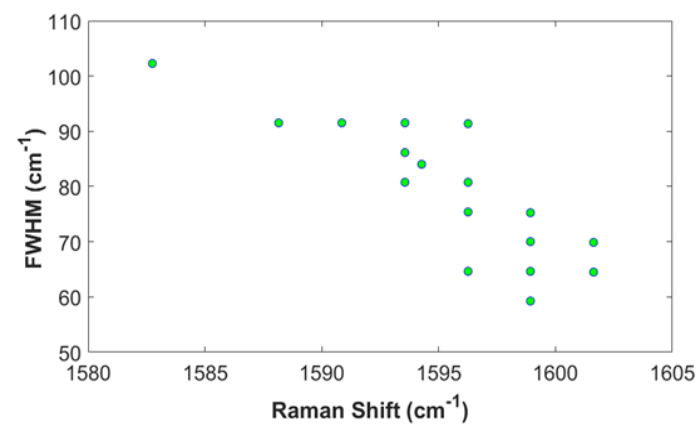

B.

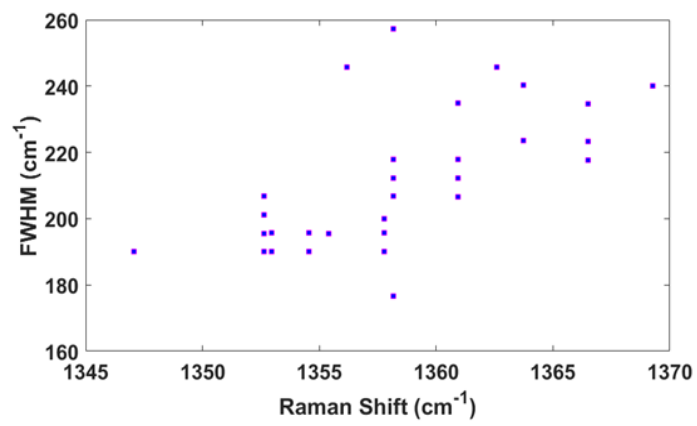

Figure 3. A. FWHM $\left(\mathrm{cm}^{-1}\right)$ vs. G-peak position. B. FWHM $\left(\mathrm{cm}^{-1}\right)$ vs. D-peak position of Al$6061 \mathrm{cv}-3 \%$ sample

Pure Al-7075, and Al-7075 cv 3\% and 5\% samples were also studied in similar manner to the Al-6061 samples. The results showed the presence of carbon in the covetic samples with similar features as in the case of the Al-6061 samples but with more variations in the map distribution. Figure $4 \mathrm{~A}$ and B show the G and D peak intensity scanned in a $4 \mu \mathrm{m} \times 4 \mu \mathrm{m}$ area to obtain better resolution with data point spacing of $500 \mathrm{~nm}$.
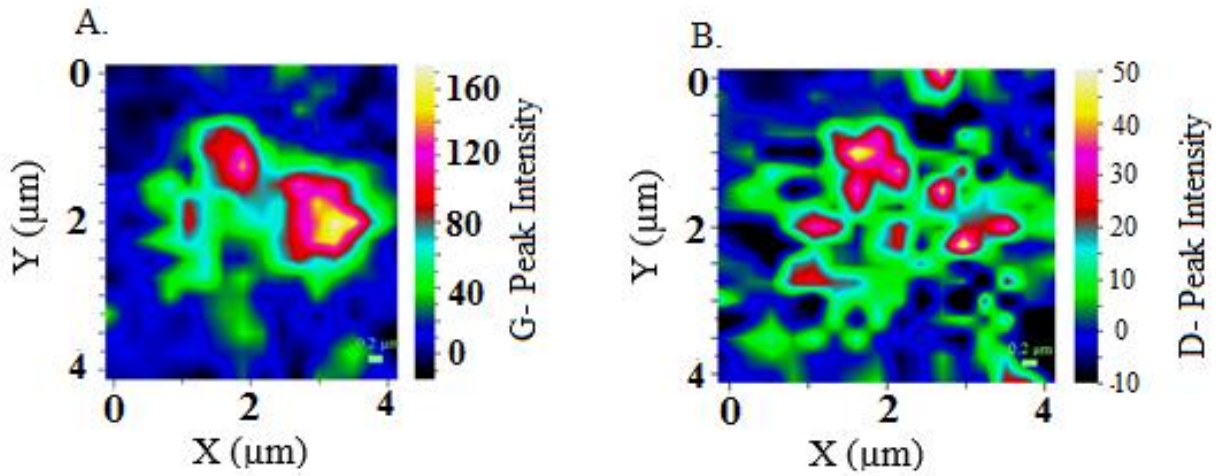

Figure 4: Raman mapping of Al-7075 cv 5\% of an area of $4 \mu \mathrm{m} \times 4 \mu \mathrm{m}$. A. G-peak intensity, B. D-peak intensity, 


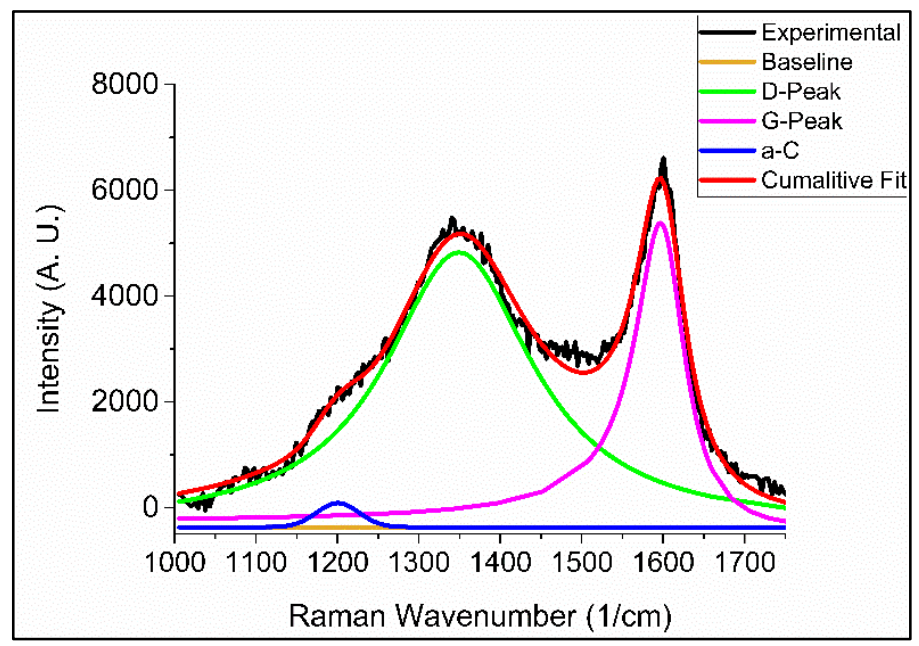

Figure 5. Raman spectrum and peak deconvolution from one of the points of Al-7075 cv 5\%. Gpeak at $1600 \mathrm{~cm}^{-1}$, D-peak at $1350 \mathrm{~cm}^{-1}$, with $\mathrm{I}_{\mathrm{D}} / \mathrm{I}_{\mathrm{G}}=2$ and an additional peak at $1200 \mathrm{~cm}^{-1}$.

Figure 5 shows the deoconvoloution of the selected regions of the Raman peaks to separate the contributions of G, D and amophous peaks. G, D and amorphous carbon peak appeared at 1600, 1350 and $1200 \mathrm{~cm}^{-1}$, respectively. Crystalline sizes of the graphitic structure in Al-7075 cv 3\% and $5 \%$ are very similar to Al-6061 cv $3 \%$.

\subsubsection{EELS of Al-6061 cv 3\%}

EELS spectrum imaging and mapping were obtained from the green rectangular area shown in the HAADF image (Figure 6 A), and magninfied in Figure 6 B from the Al-6061 cv 3\% TEM sample. The O-K edge map (Figure $6 \mathrm{C}$ ) shows slightly higher concentration of oxidation all over the surface compared to the Ag covetic sample. In the $\mathrm{C}-\mathrm{K}$ edge map (Figure $6 \mathrm{D}$ ), nanoribbon-like features of $\mathrm{C}$ are observed which correspond to the somewhat darker portion on the top of the HAADF image in Figure $6 \mathrm{~B}$. The presence of a weak $\pi^{*}$ edge at $\sim 284 \mathrm{eV}$ in the $\mathrm{C}-\mathrm{K}$ edge EELS spectrum in Figure $6 \mathrm{E}$ is indicative of $\mathrm{sp}^{2}$ carbon $[24,25]$.

BSS analysis of the EELS data in Figure 6 was carried out and is presented in Figure 7. The two dominant signals resemble the $\mathrm{C}-\mathrm{K}$ edge spectra of $\mathrm{sp}^{2}$ carbon and amorphous carbon, respectively. The highly carbon enriched region in Figure 6 is found to be due to $\mathrm{sp}^{2}$ carbon in the ribbon like regions. The amorphous carbon signal is fairly uniform as evident from the small variations in relative intensity and is believed to be due to hydrocarbon contamination from the TEM column. 
A.

B.

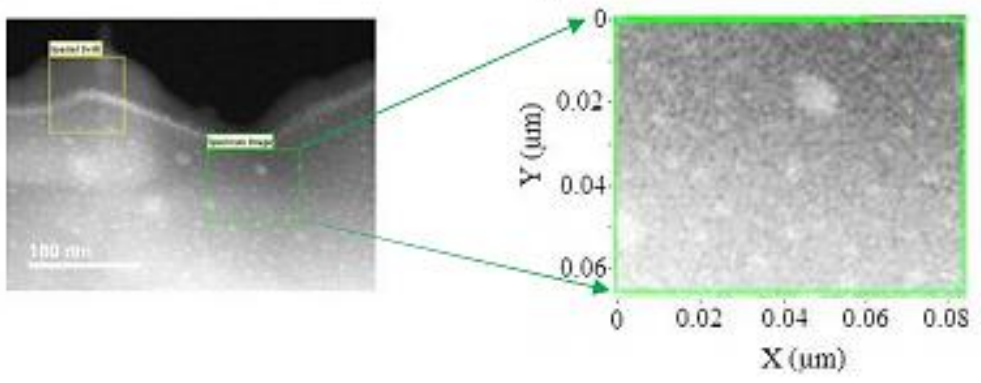

Dark Field TEM

Scanned Region

C.

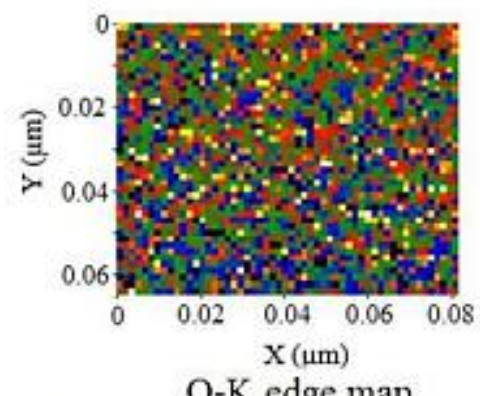

E.

D.

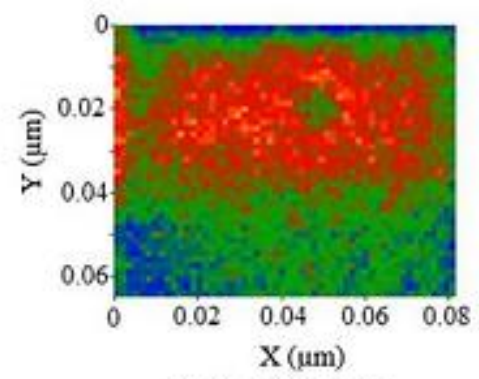

C-K edge map

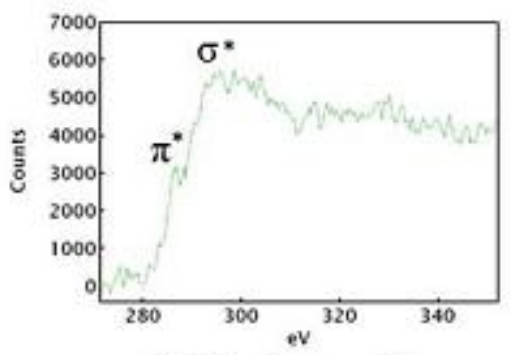

$\mathrm{C}-\mathrm{K}$ edge profile

Figure 6. A. HAADF image of Al-6061 cv 3\%. The green rectangular area labeled Spectrum Image delineates the region selected to acquire EELS spectrum imaging in the energy range for the C-K edge. The yellow rectangle (Spatial Drift) was used for EELS position calibration to offset any drift of the sample during data acquisition. B. Magnified area of the green rectangle in A. C. O-K edge map from B. D. C-K edge area map of the scanned region showing a ribbon type feature E. C-K edge profile after background subtraction from one of the points in the scanned region showing $\mathrm{sp}^{2}$ attributes. 
A.

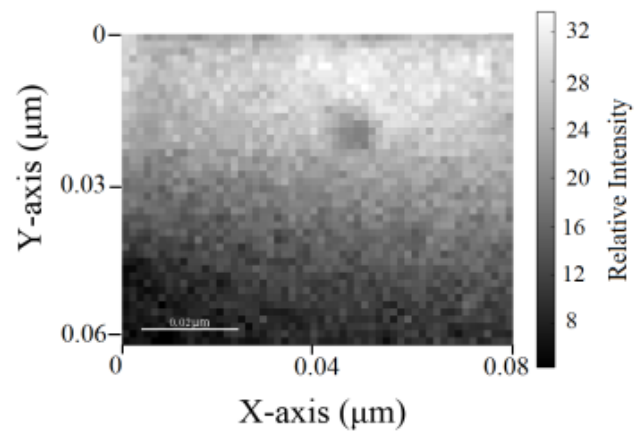

C.

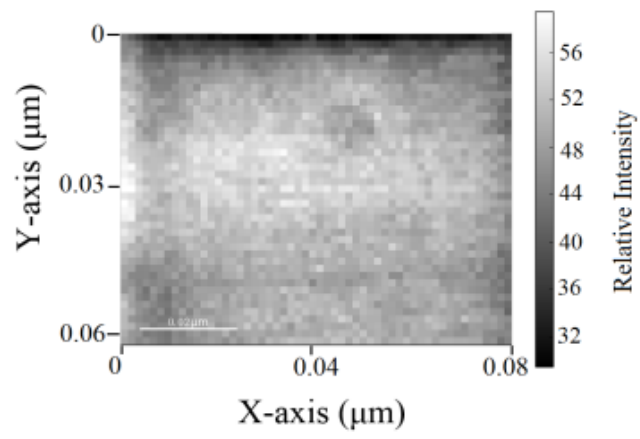

B.

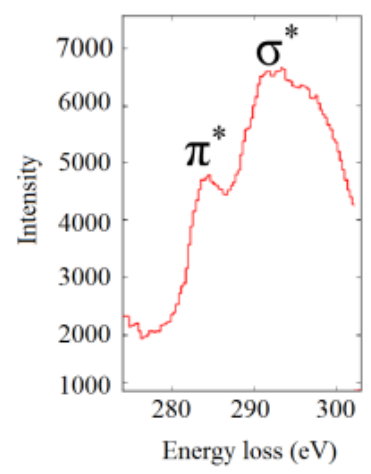

D.

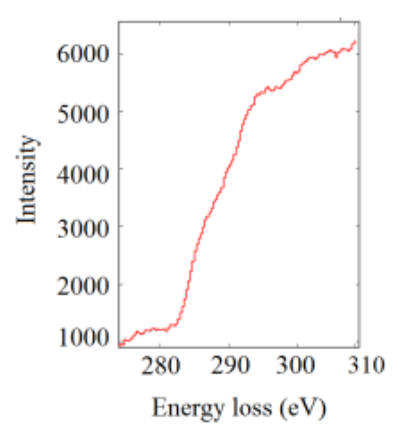

Figure 7. Spectral decomposition of the EELS mapping shown in Figure 6 by BSS. The first two primary signals and their corresponding maps are shown in the two sets. A. Map of the first signal corresponding to $\mathrm{sp}^{2}$ carbon (B) showing regions of higher intensity in ribbon form (whitish region). C. Map of the second component in the BSS analysis showing similar signal to amorphous carbon (D).

\subsubsection{Raman mapping of Ag cv $6 \%$}

Raman spectra from pure Ag and Ag cv-6\% were acquired from areas of $20 \mu \mathrm{m} \times 20 \mu \mathrm{m}$. The pure Ag did not show any carbon signal in agreement with our previous results [12]. An optical image of the scanned region of the Ag cv 6\% sample is presented in Figure $8 \mathrm{~A}$ with the points denoting where data were taken, and the regions with high intensity of the G-peak are identified. Although carbon was observed almost everywhere, the dark regions under the optical microscope showed a stronger presence of carbon apparent from the high intensity of G and Dpeaks. One representative Raman spectrum from the area is shown in Figure $8 \mathrm{~B}$ after baseline correction. The presence of broad G and D peaks, and overlapping peaks at higher wavenumbers are observed. The inset shows representative spectra of the $G$ and $D$ peaks with different intensity ratios in the same sample at three different points. 
A.

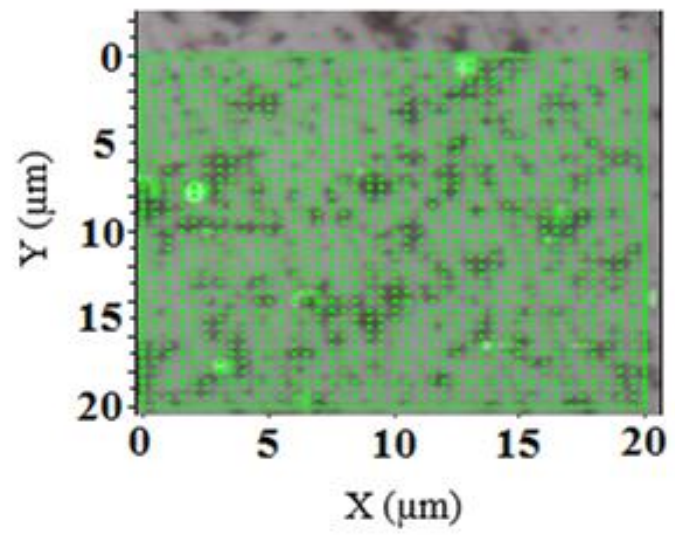

B.

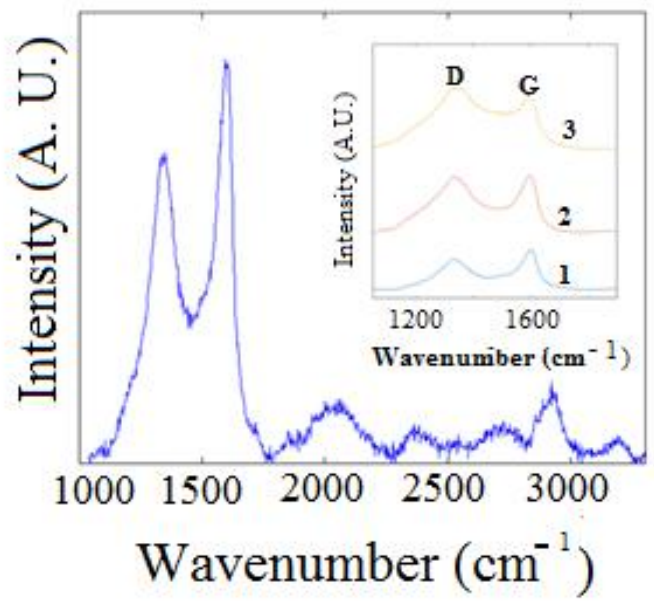

Figure 8: A. Optical image of the scanned area of a Ag-cv 6\% sample. The '+' signs show the points where spectra were obtained. The regions with high G- peak intensity are superimposed on the optical image. The contrast is set to keep the black regions visible compromising the total overlap of the G-peak intensity and the black regions. Therefore, the green regions correspond to very high carbon content in this area. B. Raman spectrum of a point from the map. The inset presents the variations in intensity and widths of the G and D peaks at three different spots of the Ag-cv 6\% sample.

Every spectrum in the scanned area in the range 1100 to $1800 \mathrm{~cm}^{-1}$ was fitted with BWF, Lorentzian and Gaussian curves for detailed analysis of the G, D and amorphous peaks, respectively. In Figure 9 two such spectra with fitted curves show the presence of G and D peaks with additional peaks arising from amorphous carbon.

A.

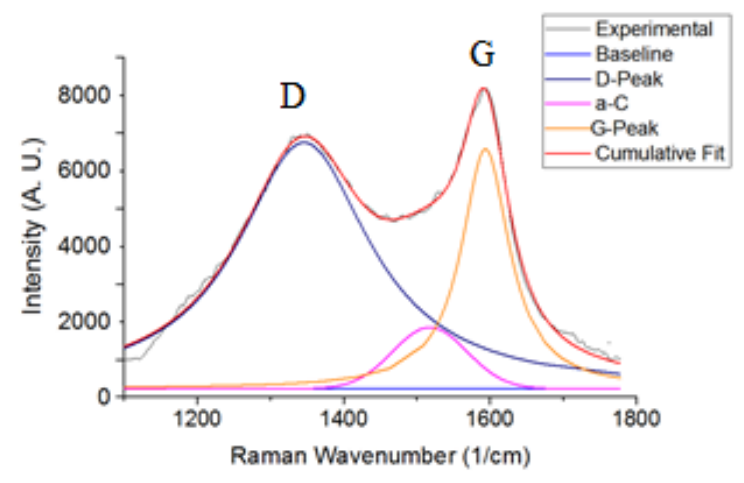

B.

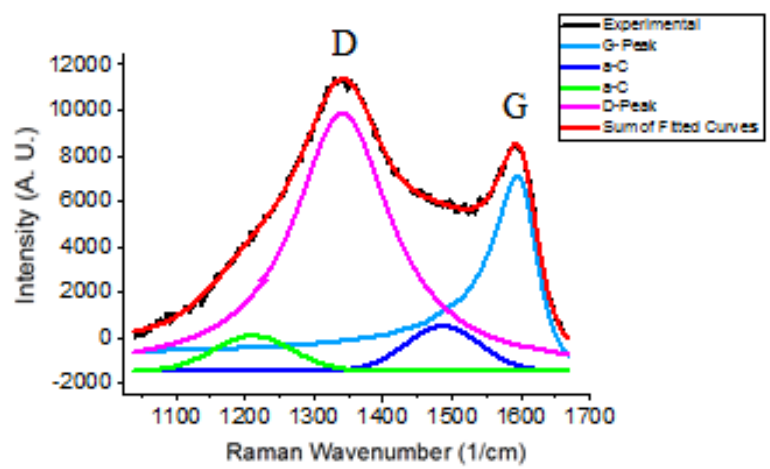

Figure 9: Deconvolution of the experimental Raman signal at two different points on Ag-cv 6\%. A. Spectrum from one point showing $G$ peak at $1599 \mathrm{~cm}^{-1}$, D peak at $1343 \mathrm{~cm}^{-1}$ and a weak additional peak at $\sim 1534 \mathrm{~cm}^{-1}$. The $\mathrm{I}_{\mathrm{D}} / \mathrm{I}_{\mathrm{G}}$ for this fitting was 2.3 . B. Spectrum from another point with $\mathrm{G}$ peak at $1596 \mathrm{~cm}^{-1}$, D peak at $1346 \mathrm{~cm}^{-1}$ and two weak additional peaks at 1211 and 1486 
$\mathrm{cm}^{-1}$. The $\mathrm{I}_{\mathrm{D}} / \mathrm{I}_{\mathrm{G}}$ was 2.4 for this fitting. The amorphous regions correspond to $\sim 10 \%$ of the integrated areas.

In Figure 10, maps of the intensity of the $G$ and D-peaks from the Ag cv $6 \%$ sample show that the regions with high $G$ peak intensity also have high $D$ peak intensity. Figure 11 presents the variations in the position of the maximum of the G-peak differentiating the strained (above $\left.\sim 1590 \mathrm{~cm}^{-1}\right)$ and unstrained $\left(\sim 1584 \mathrm{~cm}^{-1}\right)$ regions. Blue shift of the Raman spectra is observed indicating compressive strained regions. Highly strained regions (reddish regions) are surrounded by unstrained regions (greenish region color). Furthermore, strain develops gradually in cluster-like areas concentrating the highest strained regions at the center. The area percentage of the unstrained region was lower than the strained one and corresponds to the regions of higher G- and D-peak intensities. The FWHM of the G-peak varied from $50-120 \mathrm{~cm}^{-1}$ (not shown). Figure 12 show the variations of the D-peak position in the Ag cv 6\% sample confirming the presence of both $\mathrm{sp}^{2}$ and $\mathrm{sp}^{3}$ carbons with most of the region being $\mathrm{sp}^{2}$ at $\sim 1350 \mathrm{~cm}^{-1}$ (reddish regions) being surrounded by $\mathrm{sp}^{3}$ bonded carbon at $\sim 1331 \mathrm{~cm}^{-1}$ (greenish region). The variation in the FWHM of the D-peak ranged from $100 \mathrm{~cm}^{-1}$ to $300 \mathrm{~cm}^{-1}$ (not shown). The calculated crystalline sizes of the graphitic regions were 7-10 nm similar to Al-6061 covetic samples.
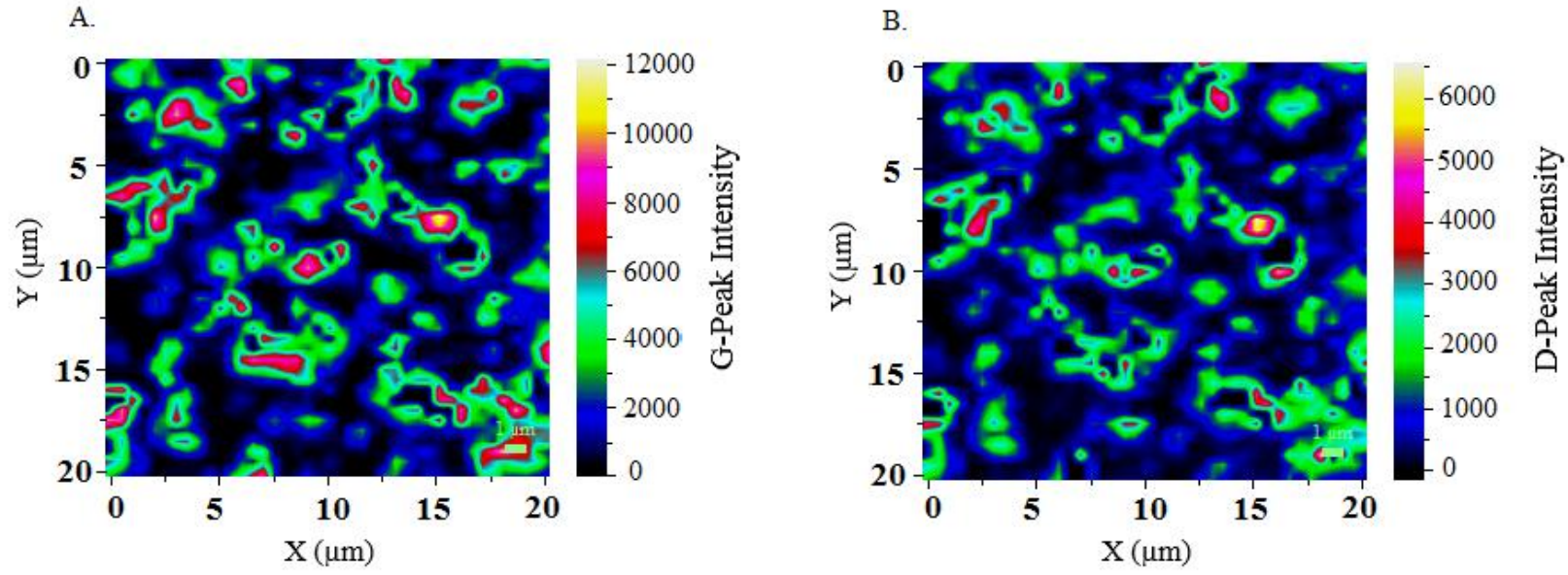

Figure 10: Raman intensity map of Ag cv 6\%. A. G-peak intensity, B. D-peak intensity.

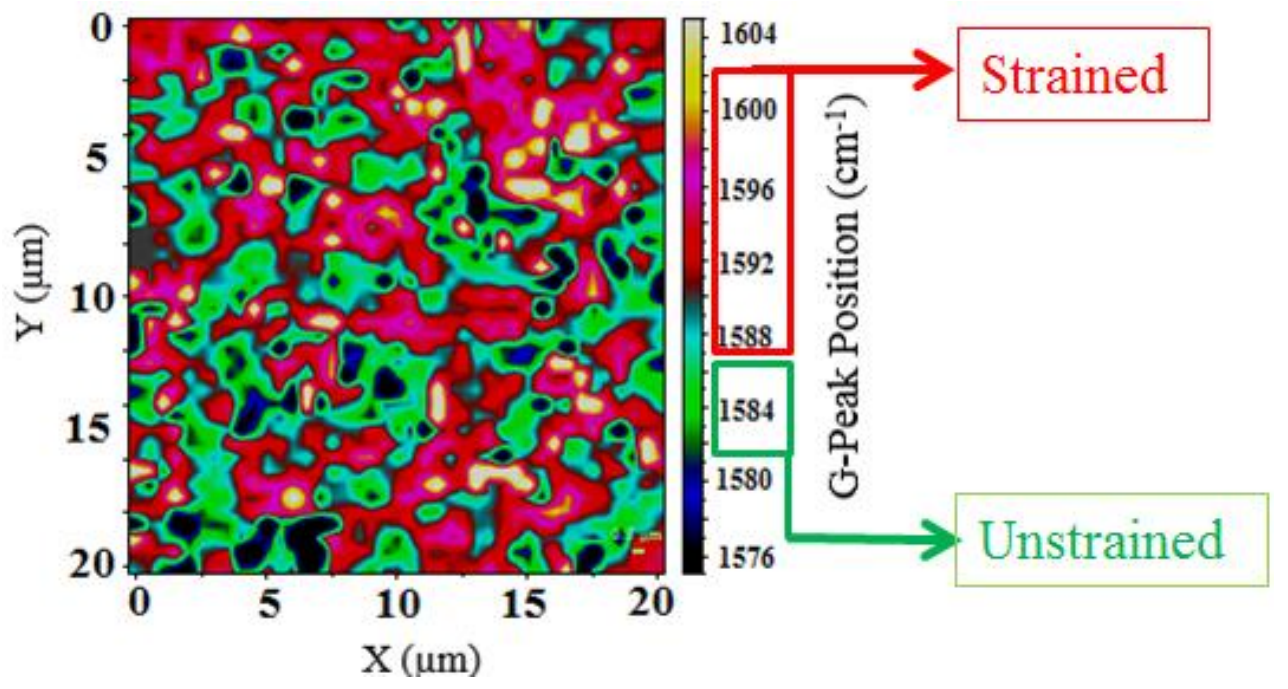


Figure 11: G-peak position variation of $\mathrm{Ag-cv} 6 \%$ ranging from $1576 \mathrm{~cm}^{-1}$ to $1606 \mathrm{~cm}^{-1}$. The greenish regions at $1585 \mathrm{~cm}^{-1}$ correspond to unstrained areas. Strain variations appear in cluster like pattern with the lower strained areas surrounding the highly strained areas.

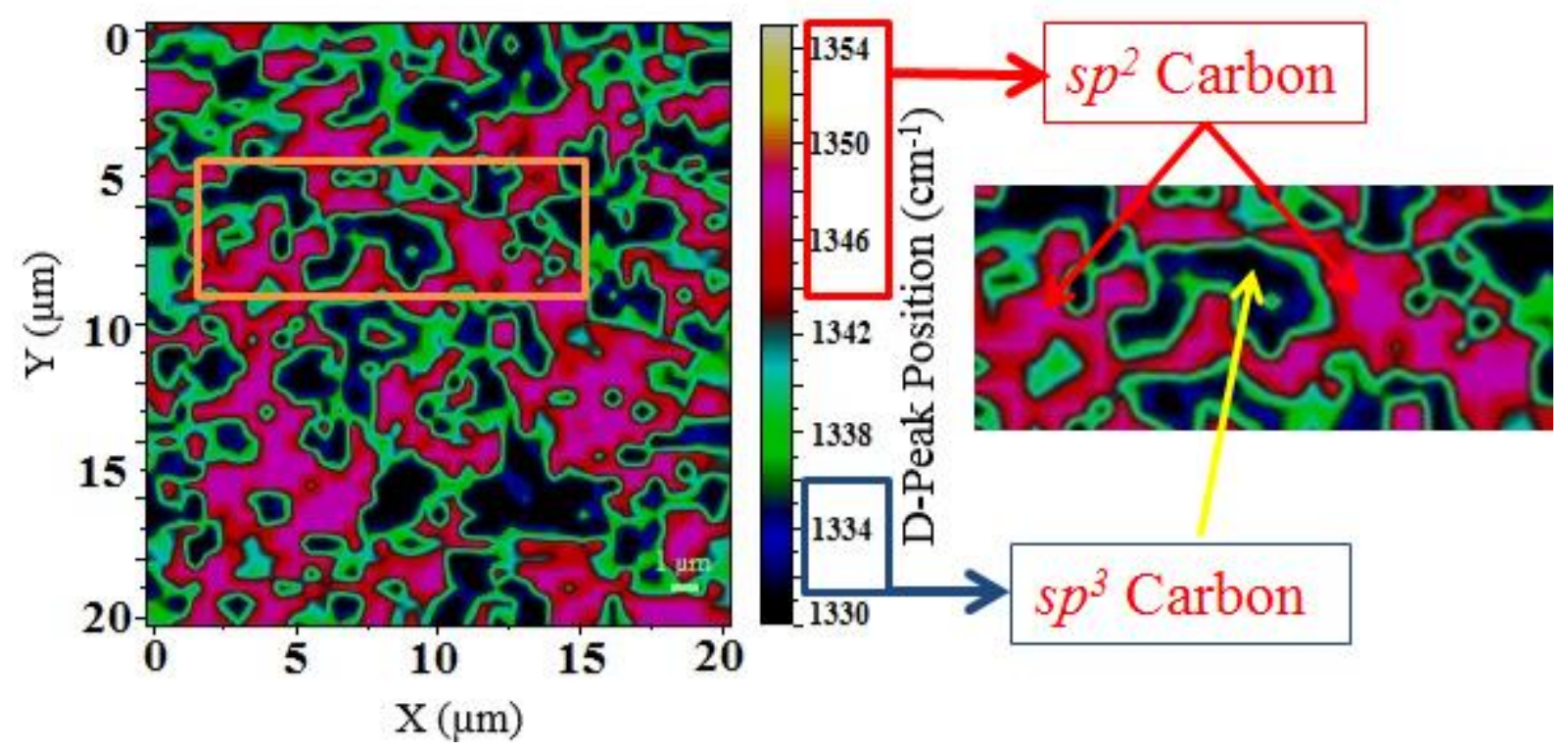

Figure 12: D-peak variation (1331 - $1354 \mathrm{~cm}^{-1}$ ) showing $\mathrm{sp}^{2}$ domain (reddish regions) network enveloped by clusters of $\mathrm{sp}^{3}$ domain (greenish regions) creating mixed bonding at the boundaries. The small rectangle area on the left is enlarged on the right.

\subsubsection{AFM and KPFM of Ag samples}

Figure 13 displays topography (A), phase (B), potential (C), and topography-potential overlay (D) maps for the pure $\mathrm{Ag}$ and $\mathrm{Ag} \mathrm{cv} 6 \%$ samples for comparison. Topographic variations were accounted for in the KPFM images through an initial forward/reverse scan, while the subsequent KPFM pass at a defined fly-height $(\sim 10 \mathrm{~nm})$ allowed the probe to map the surface potential. Dark features in the Ag cv 6\% sample are likely due to the added carbon since the tip material $(\mathrm{Pt})$ and carbon have similar work functions. Several relatively low potential areas can be seen in the $\mathrm{Ag} \mathrm{cv} 6 \%$ maps, some of which appear to form ribbon-like features. However, the exact shape of the carbon regions are difficult to discern since the scanning fly height used during KPFM results in reduced resolution and can be complicated by large variations in topography. Nevertheless, these features are not seen in the KPFM image from the pure Ag sample even though the topographic image from pure Ag shows some surface roughness. 
Pure Ag

A.

B.
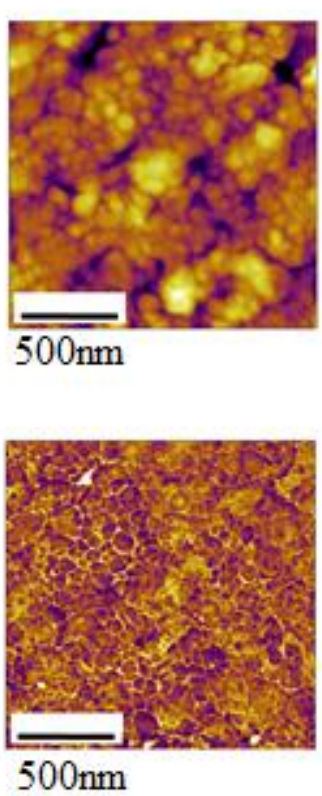

C.

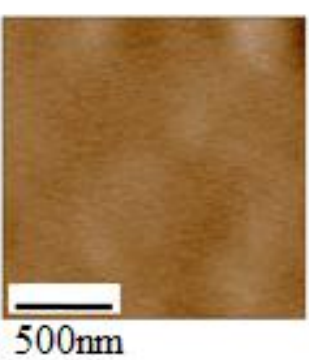

D.

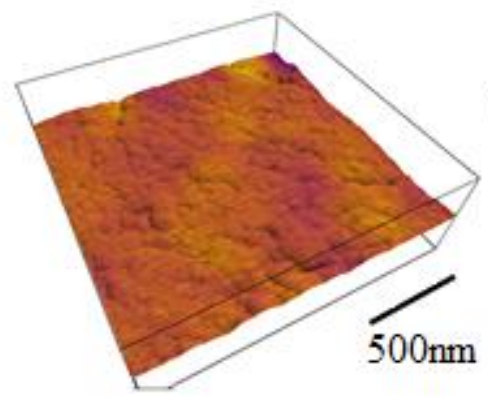

Ag cv- $6 \%$
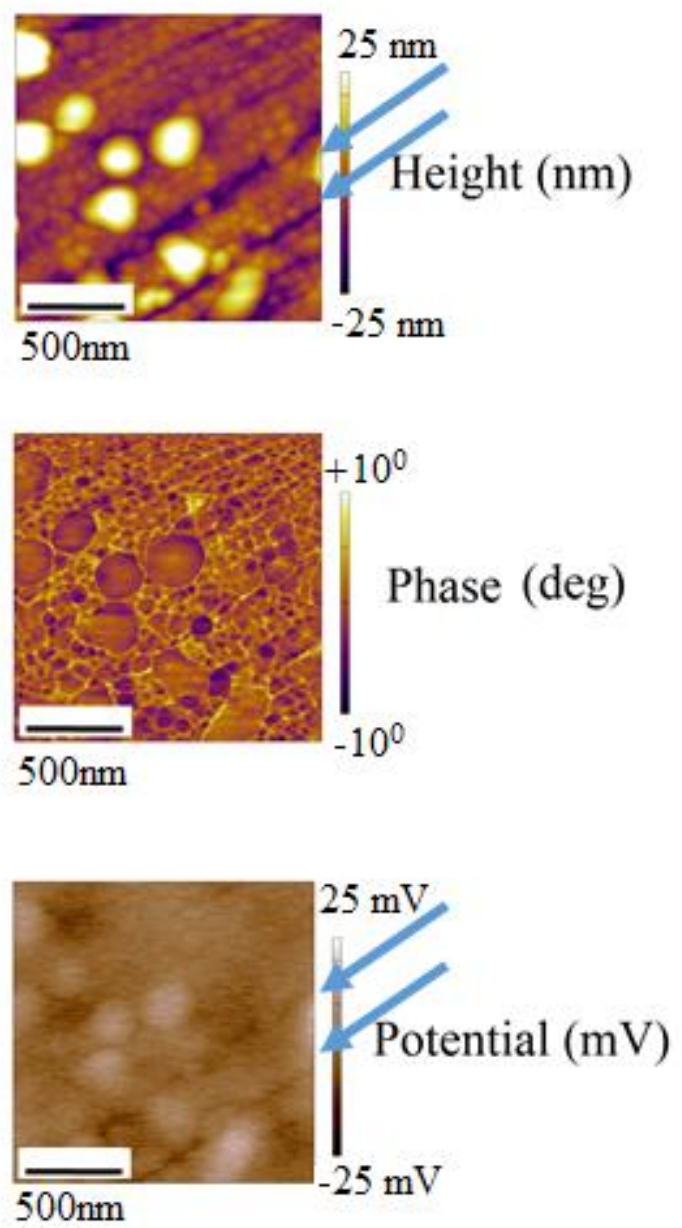

Figure 13: Comparative AFM (height and phase) and KPFM images of pure Ag and Ag cv 6\%. A. Height maps (scale: $-25 \mathrm{~nm}$ to $25 \mathrm{~nm}$ ) B. Phase maps (scale: $-10^{\circ}$ to $+10^{\circ}$ ). C. Potential maps using KPFM (scale: $-25 \mathrm{mV}$ to $25 \mathrm{mV}$ ). D. Height maps with potential overlay. Lower potential regions with phase and height contrast appear to show ribbon-type features on the bottom right regions of the covetic sample marked by blue arrows. 


\subsubsection{EELS mapping of Ag cv-6\%}

Figure 14 A shows a HAADF image from an area of the Ag-cv 6\% sample. An EELS spectrum image in the region of the $\mathrm{C}-\mathrm{K}$ edge was obtained from the area delineated by the green rectangle and magnified in Figure $14 \mathrm{~B}$. In HAADF, the lighter atoms appear as dark regions as they scatter electrons less efficiently. The darker regions in Figure $14 \mathrm{~B}$ correspond to the carbon rich zones as corroborated by the C-K edge map in Figure $14 \mathrm{C}$. Figure $14 \mathrm{D}$ presents the C-k edge profile from one of the points in Figure $14 \mathrm{C}$ showing $1 \mathrm{~s}$ to $\pi^{*}$ and $\sigma^{*}$ transitions at 284 and 290 $\mathrm{eV}$, respectively, which are the characteristic edges of graphitic carbon with $\mathrm{sp}^{2}$ bonding [24, 25]. The $\mathrm{Ag}$ concentration in this area is fairly uniform as indicated by the $\mathrm{Ag}-\mathrm{K}$ edge map in Figure $14 \mathrm{E}$. This region also contained very small amounts of oxygen as shown in the O-K edge map in Figure $14 \mathrm{~F}$ which does not seem to correlate with the regions with high carbon content.

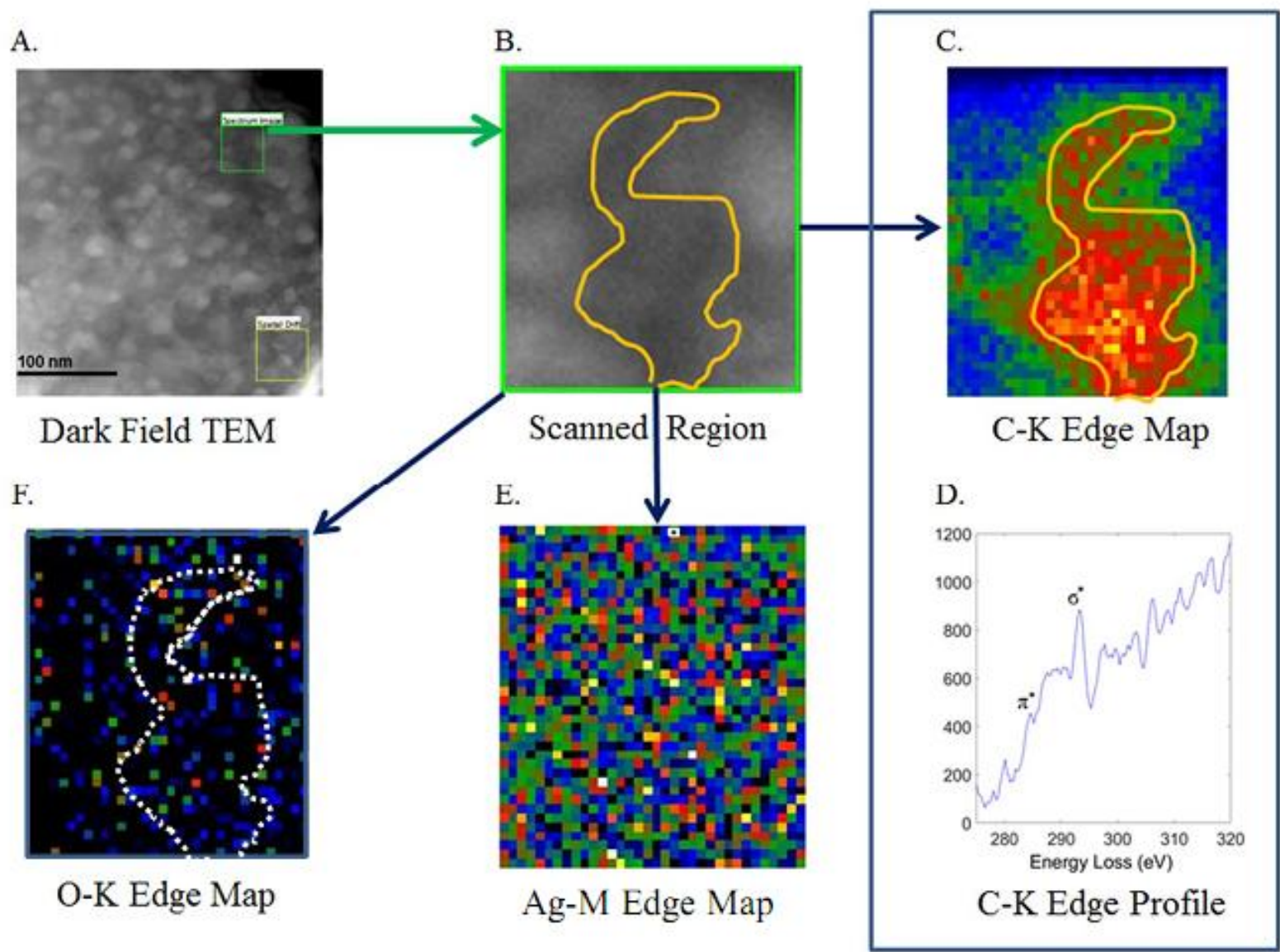

Figure 14: A. HAADF image of Ag-cv 6\%. B. Magnified region where EELS spectrum imaging corresponding to the green rectangular area on the top right region in A was obtained. C. C-K edge map in temperature gradient color. The curve encloses the region highly rich in carbon. D. $\mathrm{C}-\mathrm{K}$ edge profile from one of the points in the scanned region after background subtraction showing $\mathrm{sp}^{2}$ characteristics. E. Ag-M edge map. F. O-K edge map. 
A.

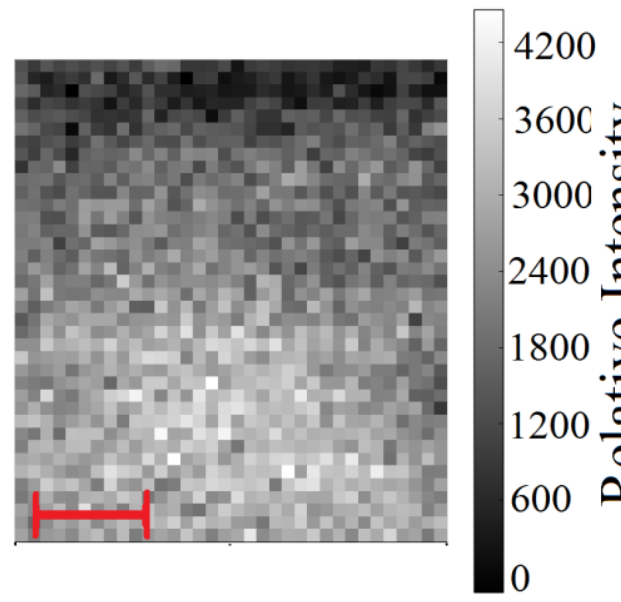

C.

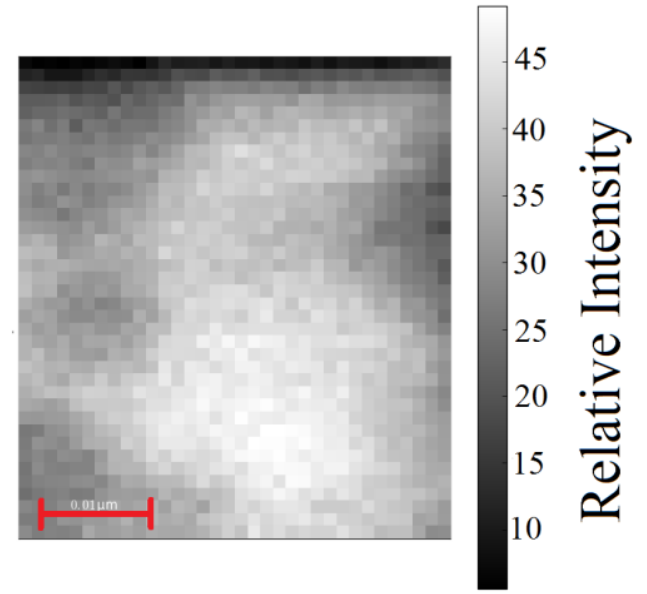

B.

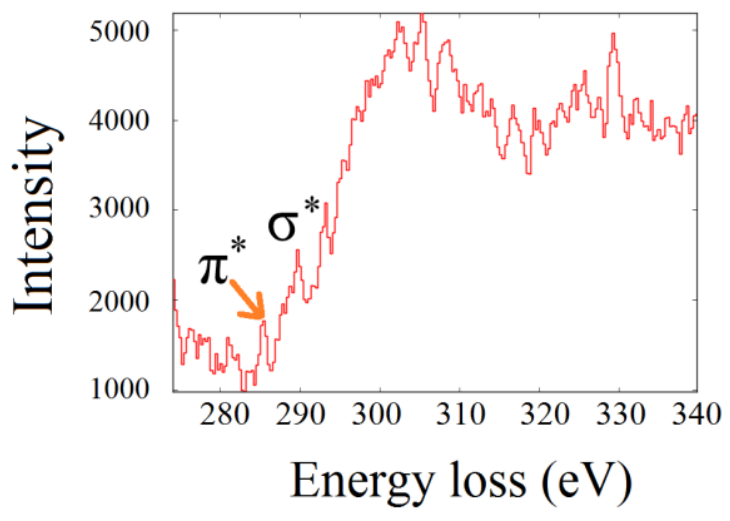

D.

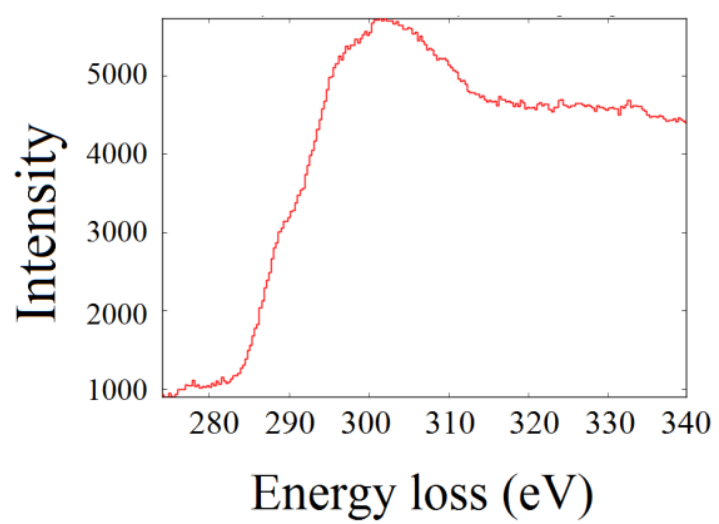

Figure 15: Spectral decomposition of the EELS mapping shown in Figure 14 by BSS method. A. A dominant signal resembling $\mathrm{sp}^{2}$ carbon shown in $\mathrm{B}$. is mapped where the high concentration is observed in the lower middle region as seen in the highly enriched carbon region in Figure $14 \mathrm{C}$. C. Another dominant signal resembling amorphous carbon signal shown in D. used for mapping. Maps of the two components in A and C coincide with the enriched carbon region in Figure 14 C. The red scale bars in A. and C. correspond to $100 \mathrm{~nm}$.

Figure $15 \mathrm{~A}$ and $\mathrm{C}$ present maps of the intensities of the signals B. and D, respectively, from the same region in Figure 14 analyzed by PCA method. The signals in Figure 15 B and D are the first and second primary components and most dominant signals in the BSS analysis and match the mapping of C-K edge in Figure $14 \mathrm{C}$. By BSS it is possible to separate the regions containing $\mathrm{sp}^{2}$ bonded carbon from the amorphous or $\mathrm{sp}^{3}$ bonded carbons that contribute to the EELS spectrum image. Thus, the images in Figure $15 \mathrm{~A}$ and $\mathrm{C}$ indicate the $\mathrm{sp}^{2}$ bonded carbon is located primarily at the bottom of the scanned region while most of the area showing strong $\mathrm{C}$ signal in Figure $14 \mathrm{C}$ corresponds to amorphous carbon. 


\section{Discussion}

All $\mathrm{Al}$ and Ag-covetic samples investigated here clearly showed the presence of $\mathrm{sp}^{2}$ carbon with varying amounts of $\mathrm{sp}^{3}$ carbon as evidenced by both Raman and EELS. However, the crystallite size and quality, degree of strain and amorphous nature of the carbon in the metal varied among the samples. Defects in the carbon are important as they contribute to the bonding with the host system [11, 12], and also influence the mechanical and electrical properties of covetics. The graphitic structures in covetics are believed to correlate with the improved global properties of the covetic samples previously observed [11-14].

In Raman mapping and spectra (Figure 1, Figure 2, Figure 4, Figure 5, Figure 9, Figure 10, Figure 11, Figure 12), all of the covetic samples showed clear G and D peaks. The intensity and width of the G-peak in graphene is dependent upon several competing factors, such as the number of layers [3], nature of carbon bonding, width of GNR, angle of bonds, and crystallinity [3]. From the Raman maps, it is obvious that there are inhomogenities present in the carbon structure, degree of graphitization, and perhaps number of graphitic layers from one region to another in all samples investigated. The relative intensity and FWHM of the D-peak depend on the concentration of defects as well as the degree of disorder in the graphitic layer. Al-covetic showed better homogeneity in terms of carbon distribution, lower strain with mostly $\mathrm{sp}^{2}$ nature of the carbon in the Raman maps (Figure 2). Comparatively, Ag covetic showed more disorder; higher degree of mixing of $\mathrm{sp}^{2}$ and $\mathrm{sp}^{3}$ bonding as evident from the higher D-peak positions (Figure 12) and their corresponding higher FWHM. The quality of carbon, quantified by the $\mathrm{I}_{\mathrm{D}} / \mathrm{I}_{\mathrm{G}}$ ratios varied from point to point in a narrow range of 2.1 to 2.5 for both $\mathrm{Al}$ and $\mathrm{Ag}$ covetic samples. Some overlap of the G and D peaks seen in individual Raman spectra (Figure 1, Figure 5, Figure 8 and Figure 9) could originate from the oxidation of the graphitic carbon as observed in the EELS spectra in Figure 6, and Figure 14 and in XPS[11, 12]. The inset to Figure 8 B shows three Raman spectra from three different points of the Ag covetic sample representing typical variations in the relative intensities and widths of the $G$ and $D$ peaks, similar to graphene oxide and reduced graphene oxide probably the result of reaction between carbon and oxygen at broken carbon bonds and at edges of graphene ribbons. Aside from the $\mathrm{G}$ and D-peaks, the $\mathrm{Ag}$ covetic (Figure 9), Al-6061 cv 3\% (Figure 1), and Al-7075 cv 5\% (Figure 5), showed peaks characteristic of amorphous carbon with maxima at different wavenumbers at $\sim 1518 \mathrm{~cm}^{-1}$ and $\sim 1200 \mathrm{~cm}^{-1}$. The peak at $1518 \mathrm{~cm}^{-1}$ was also observed in the source amorphous activated carbon (not shown), possibly originating from the hydrogenated process of production, and the size and shape of carbon clusters [26], and appears in covetic samples due to the presence of some unreacted residual carbon or carbon not fully crystallized in some regions of the samples (Figure $1 \mathrm{C}$, Figure 9). The other peak at $\sim 1200 \mathrm{~cm}^{-1}$ is known to originate from $\mathrm{sp}^{3}$ rich regions in amorphous carbon as shown in Figure 5 [26].

The absence of clear 2D peaks in all spectra, but instead overlap of weak peaks in the high wavenumber region is characteristic of GNR [27]. In case of GNR and graphene sheets, high wave number regions from 2300 to $3400 \mathrm{~cm}^{-1}$ tend to overlap, and become very broad due to the excess Raman signal produced from broken bonds, oxidized functional groups at the edges and the presence of structural defects [11]. AFM and KPFM (Figure 13) of the Ag-covetic sample, and EELS in Al-6061 covetic sample (Figure 6 and Figure 7) show the GNR-like features. Similarly, in case of Al-covetic samples, previous studies by AFM and KPFM confirmed ribbon 
like features with lower potentials [11]. Variations in the conductivity by KPFM complement the Raman data, and suggest that the carbon in covetics consists of regions with different number of layers of GNR, different levels of oxidation and defect concentration for Ag (Figure 13) and Alcovetic samples [11].

3D epitaxy of graphitic carbon in the metal matrix, and preferred direction of growth of crystalline carbon from the amorphous carbon source (activated carbon) has been confirmed for both $\mathrm{Al}$ and Ag-covetic samples from our previous TEM studies, where in addition, varying widths of ribbons were clearly observed $[11,12]$. The variations of the crystallite sizes measured by the Tuinstra-Koenigh relation from the $\mathrm{I}_{\mathrm{D}} / \mathrm{I}_{\mathrm{G}}$ ratio of the Raman spectra presented in the previous section closely match the TEM results [11]. Compressive strain was also observed in the selected area diffraction pattern (SADP) in the $\mathrm{sp}^{2}$ crystals in both $\mathrm{Ag}$ [12] and $\mathrm{Al}$ [11], and XRD showed gradual change in the lattice parameter of the Al-matrix with carbon doping. Since all covetic samples are prepared at high temperatures (above the melting temperature of the metal) to incorporate activated carbon, strains due to lattice mismatch and thermal expansion mismatch between the carbon and the metal are more likely to happen as evidenced by the shift in the position of the $\mathrm{G}$ and $\mathrm{D}$ peaks in the Raman spectra.

The position of the G-peak in graphene is at $1585 \mathrm{~cm}^{-1}$. However, there are several factors that can shift this peak to higher or lower wavenumbers. Strain is one of the factors that strongly affect the position of this peak. Reduction in crystallite size (nanocrystalline carbon) and increase of $\mathrm{sp}^{3}$ bonding also shift the position of the G-peak to higher wavenumbers [28]. In the case of uniaxial, tensile or compressive, strains in single layer graphene, red and blue shift, respectively, from the unstrained position of the G-peak have been reported [29]. The shift in the G-peak position due to strains is the result of anti-harmonic vibrational modes of the regular symmetric honeycomb structure of the $s p^{2}$ carbon due to the changes in the interatomic distances, and subsequent stiffening, or relaxation, of the bonds with a supporting substrate [30] and/or composite matrix [31]. Significant blue shift, by $10 \mathrm{~cm}^{-1}$, has been observed in graphene deposited on $\mathrm{SiC}$ substrates due to $1 \%$ compressive strain of the covalent bonds which resulted in a change of the lattice parameter [32]. Also, graphene deposited on flexible PET substrates under $1 \%$ strain produced shifts of the G-peak by $14.2 \mathrm{~cm}^{-1}$ [33]. Thermal cycling of graphene on substrates yields in-plane compression due to the mismatch of the thermal expansion coefficients between graphene and the substrate, and also contributes to a shift of the G-peak position to higher wavenumbers upon cooling the system back to room temperature [34]. Strain due to lattice mismatch between silver and graphene is the main factor giving rise to shifts of the Gpeak $[12,35]$. Shift of the G-peak, as well as suppression and shift of the 2D peak, have been reported when metal species, such as, $\mathrm{Cr}$ and $\mathrm{Ti}$ are incorporated in graphene because of the changes in electron-phonon coupling resulting from the foreign atoms.[36]. In each of these cases, the role of the substrate, number of graphene layers, and processing conditions, has an important effect on the shift of the G-peak position. For covetic samples, it is more complicated to analyze the exact origin of changes in the intensity and shift of the G-peak since covetics are not substrates with a graphene layer deposited on the surface but materials that contain graphitic structures within the bulk of the metal matrix. What we can speculate based on our previous observations of compressive strains of graphitic structures in the form of GNR and graphene sheets in $\mathrm{Ag}$ and $\mathrm{Al}$ covetics through TEM [11,12] and EELS mapping of the C-K edge in Figure 6 and Figure 14 along with evidence for Al-C and Ag-C bonding revealed by XPS and 
predicted from our DFT simulations $[11,12]$ which give rise to bending and stretching of the C$\mathrm{C}$ bonds and $\mathrm{C}$-metal bonds blue shifts of the G-peak in these samples (Figure 2 and Figure 11) are caused by the small width of GNR, which are strained in an attempt to match the low potential sites in the (111) planes of the $\mathrm{Ag}$ and $\mathrm{Al}$ host lattices during cooling to room temperature. Furthermore, the position of the G-peak also changes in graphite intercalation compounds. Since our previous studies of Ag covetic [12] show small regions of alternating (111) planes of Ag and graphene sheets, it is possible that some of the shift of the G-peak to higher wavenumbers is due to these regions. Broadening of the G-peak might also be due to bonding of the carbon with metal host atoms, through both bond bending and in plane disorder of the $C$ in the basal planes as our previous DFT calculations suggest $[11,12]$.

Variations in the position of the $\mathrm{D}$ peak were observed for both $\mathrm{Al}$ and $\mathrm{Ag}$ covetic samples. In Al-6061 cv 3\%, the D peak varied from $1367 \mathrm{~cm}^{-1}$ to $1341 \mathrm{~cm}^{-1}$ (Figure $2 \mathrm{D}$ ). The latter agrees with the position of the D peak observed at edges of monolayer graphene on the Si substrate [3739]. Regions with shift of the D peak (Figure 2 D) also had higher G-peak positions ranging from 1590-1598 $\mathrm{cm}^{-1}$. In contrast, in the Ag-covetic sample the position of the D-peak varied from 1331 to $1354 \mathrm{~cm}^{-1}$ with a network of $\mathrm{sp}^{2}$ bonded carbon being surrounded by $\mathrm{sp}^{3}$ carbon (Figure 12). The strong presence of defects as evidenced by the high intensity of the D-peak are believed to be because of the large laser spot in the Raman spectrometer $(\sim 1 \mu \mathrm{m})$ compared to the narrow width of the GNR and small (carbon) crystallite sizes $(\sim 10 \mathrm{~nm})$. Thus, the Raman spot samples many edges of GNR. $\mathrm{Sp}^{3}$ carbon appears due to amorphization as clusters of $\mathrm{sp}^{2}$ bonded carbon might be terminated by $\mathrm{sp}^{3}$ bonded carbon $[26,40,41]$. $\mathrm{Sp}^{3}$ carbon could also be due to metal-C bonding which has been observed in XPS for Al covetics and predicted by DFT simulations [11].

Lastly, mapping of the C-K edge in the EELS spectrum imaging and analysis of the EELS spectra by the BSS method confirmed the nanoribbon-like geometry in the Al-6061 cv-3\% (Figure 6 and Figure 7), and $\mathrm{Ag} \mathrm{cv} 6 \%$ (Figure 14 and Figure 15) containing primarily $\mathrm{sp}^{2}$ carbon with some mixture of $\mathrm{sp}^{3}$ carbon. The peak at $284 \mathrm{eV}$ in the EELS spectra from both samples corresponds to transitions of the $1 \mathrm{~s}$ electron in graphite to the $\pi^{*}$ antibonding unoccupied state only present in carbon with $\mathrm{sp}^{2}$ bonding $[24,25]$. Although the analyzed areas in EELS spectrum imaging are much smaller than the Raman spot, the inhomogeneity observed in Raman at microscale is better revealed in EELS mapping at the nano-scale. The presence of both $\mathrm{sp}^{2}$ and amorphous carbon in the same region seen the EELS spectrum imaging of the Ag-covetic sample after signal separation as well as Raman mapping and deconvolution of the $G$ and $D$ peaks showing both amorphous and $\mathrm{sp}^{2}$ peaks with different percentages indicates that the $\mathrm{sp}^{2}$ carbon transformation from the amorphous source of activated carbon with the application of the current in the electrocharging assisted process was not complete. Varying degrees of oxidation were observed throughout the carbon rich regions in both $\mathrm{Al}$ and $\mathrm{Ag}$-covetic samples, which agree well with previous results.

\section{Conclusion}

In this research effort, a detailed analysis of Raman spectra and EELS spectrum images of pure and covetic Ag (6 wt \% C), Al-6061 (3 wt.\% C), and Al-7075 (3 and 5 wt\% C) samples was performed to characterize the degree of graphitization produced by the application of a current in the electrocharging assisted process of covetics. This process gives rise primarily to $\mathrm{sp}^{2}$ bonding 
of the $\mathrm{C}$ atoms with small contributions of $\mathrm{sp}^{3}$ bonded carbon. Raman spectra indicate a high degree of strain in the graphitic regions and EELS mapping reveals that the carbon structures form networks of GNR in agreement with our previous TEM results. The GNR are highly defective with widths of $\sim 10 \mathrm{~nm}$. The formation of GNR was also supported by KPFM images in $\mathrm{Ag}$ covetic. Some degree of oxidation was evident primarily in the Al covetic samples since Al is prone to oxidation while $\mathrm{Ag}$ is not. Characterization of $\mathrm{sp}^{2}$ carbon embedded within metal systems is important to better understand the behavior of graphene or thin graphite in complex systems. Our results provide insight into future fabrication and characterization processes of hybrid metal-carbon, materials and have important implications in a variety of civil, aerospace, defense, and electronics applications.

\section{Acknowledgement}

This work was supported in part by ONR under contract N000141410042 and DARPA/ARL under contract W911NF13100. The Ag and Al-covetic samples were provided by Third Millennium Materials, LLC. We acknowledge the use of the TEM and SEM at the University of Maryland Nanocenter and its AIM Laboratory.

\section{References}

1 Dresselhaus, M.S., Dresselhaus, G., and Eklund, P.C.: 'Science of fullerenes and carbon nanotubes: their properties and applications', 1996, Academic Press

2 Allen, M.J., Tung, V.C., and Kaner, R.B.: 'Honeycomb carbon: a review of graphene', Chemical reviews, 2009, 110, (1), pp. 132-145

3 Dresselhaus, M.S., Jorio, A., Hofmann, M., Dresselhaus, G., and Saito, R.: 'Perspectives on carbon nanotubes and graphene Raman spectroscopy', Nano Lett, 2010, 10, (3), pp. 751-758

4 Karakaya, I., and Thompson, W.T.: 'The Ag-Pd (Silver-Palladium) system', Bulletin of Alloy Phase Diagrams, 1988, 9, (3), pp. 237-243

5 Okamoto, H., and Massalski, T.B.: 'The Au-C (Gold-Carbon) system', Bulletin of Alloy Phase Diagrams, 1984, 5, (4), pp. 378-379

6 Predel, B.: 'C-Cu (Carbon-Copper)', in Madelung, O. (Ed.): 'B-Ba - C-Zr' (Springer Berlin Heidelberg, 1992), pp. 1-1

7 Chen, J., Bi, H., Sun, S., Tang, Y., Zhao, W., Lin, T., Wan, D., Huang, F., Zhou, X., Xie, X., and Jiang, M.: 'Highly Conductive and Flexible Paper of 1D Silver-Nanowire-Doped Graphene', ACS Applied Materials \& Interfaces, 2013, 5, (4), pp. 1408-1413

8 Ogawa, F., and Masuda, C.: 'Fabrication of carbon nanofiber-reinforced aluminum matrix composites assisted by aluminum coating formed on nanofiber surface by in situ chemical vapor deposition', Materials Research Express, 2015, 2, (1), pp. 015601

9 Bastwros, M., Kim, G.-Y., Zhu, C., Zhang, K., Wang, S., Tang, X., and Wang, X.: 'Effect of ball milling on graphene reinforced Al6061 composite fabricated by semi-solid sintering', Composites Part B: Engineering, 2014, 60, pp. 111-118

10 Laha, T., Agarwal, A., McKechnie, T., and Seal, S.: 'Synthesis and characterization of plasma spray formed carbon nanotube reinforced aluminum composite', Materials Science and Engineering: A, 2004, 381, (1), pp. 249-258 
11 Jaim, H.M.I., Isaacs, R.A., Rashkeev, S.N., Kuklja, M., Cole, D.P., LeMieux, M.C., Jasiuk, I., Nilufar, S., and Salamanca-Riba, L.G.: 'Sp 2 carbon embedded in Al-6061 and Al7075 alloys in the form of crystalline graphene nanoribbons', Carbon, 2016, 107, pp. 56-66

12 Salamanca-Riba, L.G., Isaacs, Romaine A., LeMieux, Melburne C.,Wan, Jiayu,Gaskell, Karen,Jiang, Yeping,Wuttig, Manfred,Mansour, Azzam N.,Rashkeev, Sergey N.,Kuklja, Maija M.,Zavalij, Peter Y.,Santiago, Jaime R.,Hu, Liangbing: 'Synthetic Crystals of Silver with Carbon: 3D Epitaxy of Carbon Nanostructures in the Silver Lattice', Advanced Functional Materials, 2015, 25, (30), pp. 4768-4777

13 Forrest, D.R., Jasiuk, Iwona, Brown, Lloyd, Joyce, Peter, Mansour, Azzam, SalamancaRiba, Lourdes: 'Novel metal-matrix composites with integrally-bound nanoscale carbon', Nanotech 2012, 2012

14 B rown, L., Joyce, P., Forrest, D., and Wolk, J.: 'Physical and mechanical characterization of a nano carbon infused aluminum-matrix composite', Mater. Perform. Charact., 2014, 3 (1), pp. 20130023.

15 Isaacs, R.A., Zhu,H., Preston, C., Mansour, A., LeMieux, M., Zavalij, P.Y., Jaim, H.M.I., $\mathrm{Hu}$, L., and Salamanca-Riba, L.G.: 'Nanocarbon - Copper thin film as transparent electrode', Appl. Phys. Lett., 2015, 106 (19), pp. 193108

16 Jorio, A., Pimenta, M., Souza Filho, A., Saito, R., Dresselhaus, G., and Dresselhaus, M.: 'Characterizing carbon nanotube samples with resonance Raman scattering', New Journal of Physics, 2003, 5, (1), pp. 139

17 Dresselhaus, M., Jorio, A., and Saito, R.: 'Characterizing graphene, graphite, and carbon nanotubes by Raman spectroscopy', Annu. Rev. Condens. Matter Phys., 2010, 1, (1), pp. 89-108 18 Ferrari, A.C., and Robertson, J.: 'Interpretation of Raman spectra of disordered and amorphous carbon', Phys Rev B, 2000, 61, (20), pp. 14095-14107

19 Hasdeo, E.H., Nugraha, A.R., Dresselhaus, M.S., and Saito, R.: 'Breit-Wigner-Fano line shapes in Raman spectra of graphene', Phys Rev B, 2014, 90, (24), pp. 245140

20 Mazzucco ; Jan Caron ; Eric Prestat ; Gaël Donval ; Luiz Fernando Zagonel ; Michael Walls ; 'hyperspy: HyperSpy 0.8.4' (2015)

21 Bonnet, N., and Nuzillard, D.: 'Independent component analysis: A new possibility for analysing series of electron energy loss spectra', Ultramicroscopy, 2005, 102, (4), pp. 327-337

22 Bosman, M., Watanabe, M., Alexander, D.T.L., and Keast, V.J.: 'Mapping chemical and bonding information using multivariate analysis of electron energy-loss spectrum images', Ultramicroscopy, 2006, 106, (11-12), pp. 1024-1032

23 Tuinstra, F., and Koenig, J.L.: 'Raman Spectrum of Graphite', J Chem Phys, 1970, 53, (3), pp. 1126-1131

24 Suenaga, K., and Koshino, M.: 'Atom-by-atom spectroscopy at graphene edge', Nature, 2010, 468, (7327), pp. 1088-1090

25 Egerton, R., and Whelan, M.: 'Electron energy loss spectra of diamond, graphite and amorphous carbon', Journal of Electron Spectroscopy and Related Phenomena, 1974, 3, (3), pp. 232-236

26 Schwan, J., Ulrich, S., Batori, V., Ehrhardt, H., and Silva, S.: 'Raman spectroscopy on amorphous carbon films', Journal of Applied Physics, 1996, 80, (1), pp. 440-447

27 Shimizu, T., Haruyama, J., Marcano, D.C., Kosinkin, D.V., Tour, J.M., Hirose, K., and Suenaga, K.: 'Large intrinsic energy bandgaps in annealed nanotube-derived graphene nanoribbons', Nat Nanotechnol, 2011, 6, (1), pp. 45-50 
28 Yang, X., Li, J., Zhou, Z., Wang, Y., Yang, L., Zheng, W., and Sun, C.Q.: 'Raman spectroscopic determination of the length, strength, compressibility, Debye temperature, elasticity, and force constant of the $\mathrm{C}-\mathrm{C}$ bond in graphene', Nanoscale, 2012, 4, (2), pp. 502-510 29 Mohiuddin, T., Lombardo, A., Nair, R., Bonetti, A., Savini, G., Jalil, R., Bonini, N., Basko, D., Galiotis, C., Marzari, N., Novoselov, K., Geim, A., and Ferrari, A.: 'Uniaxial strain in graphene by Raman spectroscopy: G peak splitting, Grüneisen parameters, and sample orientation', Phys Rev B, 2009, 79, (20)

30 Das, A., Chakraborty, B., and Sood, A.K.: 'Raman spectroscopy of graphene on different substrates and influence of defects', B Mater Sci, 2008, 31, (3), pp. 579-584

31 Hadjiev, V.G., Iliev, M.N., Arepalli, S., Nikolaev, P., and Files, B.S.: 'Raman scattering test of single-wall carbon nanotube composites', Applied Physics Letters, 2001, 78, (21), pp. 3193-3195

32 Wang, Y.Y., Ni, Z.H., Yu, T., Shen, Z.X., Wang, H.M., Wu, Y.H., Chen, W., and Shen Wee, A.T.: 'Raman studies of monolayer graphene: the substrate effect', The Journal of Physical Chemistry C, 2008, 112, (29), pp. 10637-10640

33 Ni, Z.H., Yu, T., Lu, Y.H., Wang, Y.Y., Feng, Y.P., and Shen, Z.X.: 'Uniaxial Strain on Graphene: Raman Spectroscopy Study and Band-Gap Opening', Acs Nano, 2008, 2, (11), pp. 2301-2305

34 Chen, C.C., Bao, W.Z., Chang, C.C., Zhao, Z., Lau, C.N., and Cronin, S.B.: 'Raman spectroscopy of substrate-induced compression and substrate doping in thermally cycled graphene', Phys Rev B, 2012, 85, (3)

35 Wang, W.X., Liang, S.H., Yu, T., Li, D.H., Li, Y.B., and Han, X.F.: 'The study of interaction between graphene and metals by Raman spectroscopy', Journal of Applied Physics, 2011, 109, (7), pp. 07C501

36 Iqbal, M., Singh, A.K., Iqbal, M., and Eom, J.: 'Raman fingerprint of doping due to metal adsorbates on graphene', Journal of Physics: Condensed Matter, 2012, 24, (33), pp. 335301

37 Yang, X.X., Li, J.W., Zhou, Z.F., Wang, Y., Yang, L.W., Zheng, W.T., and Sun, C.Q.: 'Raman spectroscopic determination of the length, strength, compressibility, Debye temperature, elasticity, and force constant of the C-C bond in graphene', Nanoscale, 2012, 4, (2), pp. 502-510

38 Gupta, A.K., Russin, T.J., Gutiérrez, H.R., and Eklund, P.C.: 'Probing Graphene Edges via Raman Scattering', Acs Nano, 2009, 3, (1), pp. 45-52

39 Graf, D., Molitor, F., Ensslin, K., Stampfer, C., Jungen, A., Hierold, C., and Wirtz, L.: 'Spatially resolved raman spectroscopy of single- and few-layer graphene', Nano Lett, 2007, 7, (2), pp. 238-242

40 Pimenta, M.A., Dresselhaus, G., Dresselhaus, M.S., Cancado, L.G., Jorio, A., and Saito, R.: 'Studying disorder in graphite-based systems by Raman spectroscopy', Physical chemistry chemical physics : PCCP, 2007, 9, (11), pp. 1276-1291

41 Chen, Z.Y., Zhao, J.P., Yano, T., Ooie, T., Yoneda, M., and Sakakibara, J.: 'Observation of $\mathrm{sp}^{3}$ bonding in tetrahedral amorphous carbon using visible Raman spectroscopy', Journal of Applied Physics, 2000, 88, (5), pp. 2305-2308 